Middle cerebral artery responses to vasoreactivity protocols

1

2

3

\title{
Protocol-dependence of middle cerebral artery dilation to modest hypercapnia
}

\author{
Baraa K. Al-Khazraji ${ }^{1}$, Sagar Buch ${ }^{3}$, Mason Kadem ${ }^{4}$, Brad J. Matushewski ${ }^{2}$ Kambiz Norozi ${ }^{5,8}$, \\ Ravi S. Menon ${ }^{3,6}$, and J. Kevin Shoemaker ${ }^{2,7}$
}

${ }^{1}$ Department of Kinesiology, Faculty of Science, McMaster University, Hamilton, ON;

${ }^{2}$ School of Kinesiology, Faculty of Health Sciences;

${ }^{3}$ Centre for Functional and Metabolic Mapping, Robarts Research Institute;

${ }^{4}$ School of Biomedical Engineering, McMaster University, Hamilton, ON;

${ }^{5}$ Department of Pediatrics, ${ }^{6}$ Department of Medical Biophysics, ${ }^{7}$ Department of

Physiology and Pharmacology, Schulich School of Medicine and Dentistry, Western University,

\section{Correspondence}

J. Kevin Shoemaker, $\mathrm{PhD}$

5 The University of Western Ontario, London, Ontario, Canada, N6A 5K7

Email:kshoemak@uwo.ca

Phone: 519-661-2111, ext: 88157 
Middle cerebral artery responses to vasoreactivity protocols

\section{Abstract}

29 There is a need for improved understanding of how different cerebrovascular reactivity (CVR)

30 protocols affect vascular cross-sectional area (CSA) when measures of vascular CSA are not

31 feasible. In human participants, we delivered $\sim \pm 4 \mathrm{mmHg}$ end-tidal partial pressure of $\mathrm{CO}_{2}$

$32\left(\mathrm{PETCO}_{2}\right)$ relative to baseline through controlled delivery, and measured changes in middle

33 cerebral artery (MCA) cross-sectional area (CSA; magnetic resonance imaging (7 Tesla MRI)),

34 blood velocity (transcranial Doppler and Phase contrast MRI), and calculated CVR based on 35 steady-state versus a ramp protocol during two protocols: a 3-minute steady-state $(+4 \mathrm{mmHg}$ $36 \mathrm{PETCO}_{2}$ ) and a ramp (delta of -3 to $+4 \mathrm{mmHg}$ of $\mathrm{PETCO}_{2}$ ). We observed that 1) the MCA did not 37 dilate during the ramp protocol, but did dilate during steady-state hypercapnia, and 2) MCA blood 38 velocity CVR was similar between ramp and steady-state hypercapnia protocols, although 39 calculated MCA blood flow CVR was greater during steady-state hypercapnia than during ramp, 40 the discrepancy due to MCA CSA changes during steady-state hypercapnia. Due to the ability to 41 achieve similar levels of MCA blood velocity CVR as steady-state hypercapnia, the lack of change 42 in MCA cross-sectional area, and the minimal expected change in blood pressure, we propose that 43 a ramp model, across a delta of $\sim-3$ to $+4 \mathrm{mmHg} \mathrm{PETCO}_{2}$, may provide one alternative approach 44 to collecting CVR measures in young adults with TCD when CSA measures are not feasible.

Keywords

47 cerebrovascular reactivity, hypercapnia protocols, transcranial Doppler, magnetic resonance

48 imaging, middle cerebral artery dilation

\section{Running Title}

51 Middle cerebral artery responses to vasoreactivity protocols 
Middle cerebral artery responses to vasoreactivity protocols

\section{Introduction}

53 Cerebrovascular reactivity (CVR) studies assess changes in cerebral blood flow to a known 54 vasoreactive stimulus (e.g., changes in end-tidal partial pressure of $\mathrm{CO}_{2} ; \mathrm{PETCO}_{2}$ ). Measures of 55 CVR are important because attenuated CVR may reflect preclinical vascular pathophysiology and 56 an increased risk of mortality independent from cardiovascular risk factors or stroke incidence (1).

57 The most commonly used technique for CVR measures in humans, transcranial Doppler (TCD) 58 ultrasonography, provides an index of vascular blood flow changes (i.e., blood velocity) because 59 the vascular cross-sectional area (CSA) values required for blood flow calculations (i.e., the 60 product of CSA and blood velocity) are not collected with TCD. Thus, an assumption of an 61 unchanging CSA is typically accepted, raising concern if changes in CSA do occur (2). To 62 circumvent issues related to TCD measures of CVR, some research groups measure all four brain63 supply (i.e., carotid and vertebral) arteries outside of the brain (3), or use expensive neuroimaging approaches $(4,5)$.

65 An additional concern regarding quantification of CVR is the potential for changes in central 66 hemodynamics during hypercapnia (elevated $\mathrm{PETCO}_{2}$ ) that could elevate cerebral blood flow due 67 to changes in cardiac output (6) and blood pressure $(6,7)$ and not directly due to cerebrovascular 68 dilation (7). Another complicating factor in quantifying CVR between groups is potential variation 69 in large cerebral artery reactivity, particularly when comparing age differences (8). As an example, 70 our group's previous work showed that compared to younger adults, older adults exhibited 71 attenuated changes in large cerebral artery CSA in response to steady-state hypercapnia (9). 72 However, obtaining cerebral artery CSA data requires access to costly MRI or CT systems. We 73 aim to understand protocol designs that provide accurate CVR estimates when using TCD 74 methods. The "ideal" velocity based CVR protocols conducted using TCD would require: 1) 75 minimal CSA changes by conducting CVR protocols that result in negligible change in CSA, and 76 2) minimal influence of confounding variables such as blood pressure.

77 In the current study, we tested the hypothesis that a ramp (i.e., linear) CVR protocol within the \pm 5 $78 \mathrm{mmHg}$ range of relative changes in $\mathrm{PETCO}_{2}$ would provide minimal changes in CSA while still 79 replicating CVR outcomes from the more standard steady-state hypercapnia CVR protocol. Our 80 rationale for this range of relative changes in $\mathrm{PETCO}_{2}$ comes from the emerging knowledge of a 81 sigmoidal change in MCA CSA, with minimal changes in hypercapnia, within the -5 to $+5 \mathrm{mmHg}$ 
Middle cerebral artery responses to vasoreactivity protocols

82 from resting $\mathrm{PETCO}_{2}$ (10). We chose a ramp-style CVR protocol to compare to CVR measures

83 from a steady-state hypercapnia protocol ( 0 to $\sim 5 \mathrm{mmHg}$ ) because: 1$)$ the ramp protocol is a well-

84 established protocol for CVR measures $(7,11,12)$, and 2) existing means for calculating CVR from

85 standard steady-state protocols use linear slope methods which simply reduce to a ramp design.

86 We acknowledge that the ramp protocol within the $\sim \Delta \pm \mathrm{mmHg}$ from baseline $\mathrm{PETCO}_{2}$ range may

87 potentially affect vascular dilation differently than a steady-state hypercapnia protocol as the

88 hypocapnia portion preceding hypercapnia may blunt blood velocity CVR (13). A sub-analysis in

89 our earlier work, however, indicated that order of condition did not affect CSA reactivity in young

90 adults (4). Additionally, existing methods primarily focus on velocity-based CVR without

91 considering CSA, and we wanted to design a CVR protocol that minimized CSA changes (even if

92 it involved hypocapnia) and blood pressure changes while retaining its ability to elevate cerebral

93 blood flow (i.e., changing blood velocity). Other models can be considered but we want to test the

94 ramp protocol as one example of alternative CVR designs that may elicit negligible CSA and BP

95 changes when measuring blood velocity CVR using TCD.

96 To achieve the high temporal resolution of the MCA CSA for the required study, we developed a

97 dynamic anatomical imaging sequence with high-temporal and spatial resolution to capture MCA

98 CSA changes every 14 seconds throughout each vasoreactivity protocol $(\sim \pm \Delta 4 \mathrm{mmHg})$ using $7 \mathrm{~T}$

99 magnetic resonance imaging. Our objectives were to assess whether between steady-state and ramp

100 protocols: 1) the MCA CSA increased compared to baseline, 2) blood pressure remained stable

101 throughout duration of the protocol, and 3) MCA blood velocity (via TCD alone), and calculated

102 flow vasoreactivity were similar.

\section{Materials and Methods}

\section{Participants}

105 All testing was conducted at the Centre for Functional and Metabolic Mapping at The University 106 of Western Ontario. The Human Subjects Research Ethics Board at the University of Western 107 Ontario (London, Ontario, Canada) approved the experiment protocols herein. Informed consent 108 from 12 healthy subjects (19-25 years of age; 6 males) was obtained prior to scanning. A sample 109 size calculation was based off of our previous work with blood velocity reactivity measured with 
Middle cerebral artery responses to vasoreactivity protocols

110 TCD during steady-state hypercapnia (4). Specifically, for a within-subject design, with a Cohen's

$111 d$ of 1.02, alpha level of significance of 0.05 , and statistical power of 0.80 , we calculated a sample

112 size of 10 and recruited 12 individuals due to our laboratory's expected attrition rate of 10-12\%

113 with our neuroimaging studies. Participants were ineligible if they were smokers, pregnant, or had

114 any of the following conditions: Raynaud's disease, respiratory illnesses, diabetes, claustrophobia,

115 history of psychosis, eating disorders, manic or bipolar disorder, major psychiatric conditions, or

116 dependence on alcohol or drugs.

\section{Procedure and data recording}

118 Testing was completed between 10am - 2pm. Participants refrained from exercise, alcohol, drugs, 119 and caffeine within 12 hours prior to testing. We used TCD and MRI to assess the cerebral 120 vasoreactivity in response to steady-state (three minutes) bouts of hypercapnia (HC), and a ramp 121 protocol from hypocapnia to hypercapnia (four minutes) (Fig. 1).
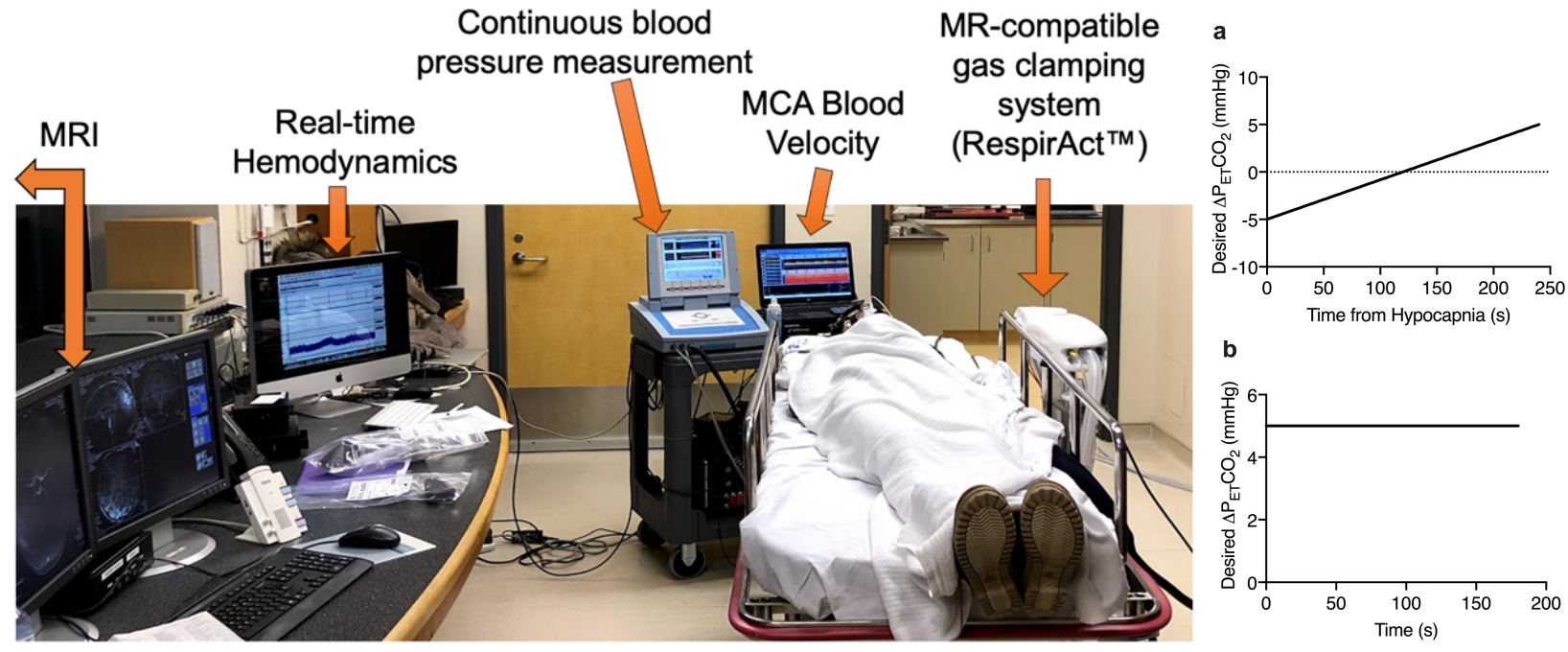

Figure 1 - Experimental protocol schematic. Left: Experimental setup displaying real-time data collection for both transcranial Doppler and MRI sessions. Right, panel a: Ramp hypercapnia protocol with a desired target range of -5 to $+5 \mathrm{mmHg}$ from baseline $\mathrm{PETCO}_{2}$ and a duration of 240 seconds. Right, panel b: Steady-state hypercapnia (SSHC) protocol with a target range of $+5 \mathrm{mmHg}$ from baseline $\mathrm{PETCO}_{2}$ and a duration of 180 seconds. Protocols were executed using the RespirAct ${ }^{\mathrm{TM}}$ device with preset protocols programmed with the desired $\mathrm{PETCO}_{2}$ values.

122 Steady-state and ramp protocols were each conducted twice, once for each of the TCD and MRI 123 portions of the testing sessions and the order of TCD and MRI trials was randomized across

124 participants. Unfortunately, we were unable to randomize the order of CVR protocols as it was 125 difficult to stop and restart the RespirAct ${ }^{\mathrm{TM}}$ (Thornhill Research Inc., Toronto, Ontario, Canada) 
Middle cerebral artery responses to vasoreactivity protocols

126 without doing extensive recalibration. The desired ventilatory rate was set to 12 breaths/min using

127 a visual metronome for each session and was projected on a screen during the MRI scan. Our goal

128 was to have the protocols fall within the $\pm 5 \mathrm{mmHg}$ from baseline $\mathrm{PETCO}_{2}$ range. Following a

129 familiarization period of four minutes, the order and duration of protocols occurred as follows: 1)

130 baseline (1 minute), 2) steady-state hypercapnia (target was $+5 \mathrm{mmHg}$, although only reached

$131 \sim+4 \mathrm{mmHg}$; three minutes), 3) recovery (2 minutes) 4) baseline (1 minute), 5) -5 mmHg $\mathrm{PETCO}_{2}$

132 hypocapnia (brief hyperventilation; target was $+5 \mathrm{mmHg}$, although only reached $\sim-3 \mathrm{mmHg}) 30$

133 seconds), 6) incremental increase (ramp) from $\sim-3 \mathrm{mmHg}$ hypocapnia to $\sim+4 \mathrm{mmHg}$ relative

$134 \mathrm{PETCO}_{2}$ hypercapnia (four minutes), 7) recovery (two minutes).

\section{Manipulating target PETCO $\mathrm{O}_{2}$ stimulus}

136 Prior to the MRI scan, participants were fitted with a facemask attached to the RespirAct ${ }^{\mathrm{TM}}$ system, 137 a modified sequential gas delivery breathing circuit(13) was used to clamp PETCO $_{2}$ levels at the 138 desired +5 or $-5 \mathrm{mmHg}$ (depending on protocol). Breathing rate and tidal volumes were calibrated 139 prior to starting the breathing sequence.

\section{$140 \quad$ MCA blood velocity and systemic blood pressure}

141 While supine, continuous beat-to-beat arterial blood pressure was monitored using a Finapres ${ }^{\circledR}$

142 Finometer system, where a finger cuff was placed on the middle phalange of the third finger, and

143 the finger blood pressure was calibrated with an upper arm cuff (Finapres ${ }^{\circledR}$ Medical Systems, 144 Amsterdam, Netherlands). The MCA was insonated with a $2 \mathrm{MHz}$ ultrasound probe placed at the 145 temporal window and the peak blood flow velocity envelope was collected using the Neurovision 146 TCD System (Multigon Industries Inc., NY, USA). All analog data were sampled at $1000 \mathrm{~Hz}$ using 147 the PowerLab data acquisition system (ADInstruments, Dunedin, Otago, New Zealand).

\section{MCA vascular diameter and blood velocity during MRI}

149 A 7 Tesla MRI (Siemens, Magnetom Step 2.3, Erlangen, Germany) system was used to acquire 150 the following datasets: 1) 3D time-of-flight (TOF) with $0.8 \mathrm{~mm}$ isotropic voxel resolution, echo 151 time $(\mathrm{TE}) /$ repetition time $(\mathrm{TR})=2.59 \mathrm{~ms} / 18 \mathrm{~ms}$, flip angle $(\mathrm{FA})=15^{\circ}$, bandwidth $(\mathrm{BW})=$ 152 203Hz/pxl; 2) single-slice 2D phase contrast (PC-MRI) for MCA M1 segment blood velocity, with 153 a voxel resolution of $0.3 \times 0.3 \times 1.4 \mathrm{~mm}^{3}, \mathrm{TE} / \mathrm{TR}=7.72 \mathrm{~ms} / 24.3 \mathrm{~ms}$, four averages, FA $=20^{\circ}$, 154 velocity-encoding $($ Venc $)=100 \mathrm{~cm} / \mathrm{s}$ and $\mathrm{BW}=250 \mathrm{~Hz} / \mathrm{pxl}$. During PC-MRI, a Venc value of 
Middle cerebral artery responses to vasoreactivity protocols

$155100 \mathrm{~cm} / \mathrm{s}$ was used for all subjects, except for one hypercapnic case, where a Venc of $130 \mathrm{~cm} / \mathrm{s}$ was 156 used to avoid wrap-around artifact; and 3) cross-sectional area of the MCA M1 segment using 157 single-slice 2D turbo spin-echo T1-weighted imaging with $0.5 \times 0.5 \times 1.5 \mathrm{~mm}^{3}, \mathrm{TE} / \mathrm{TR}=12 / 750 \mathrm{~ms}$, $158 \mathrm{BW}=270 \mathrm{~Hz} / \mathrm{pxl}$, with an acquisition time of 13-14 seconds. The TOF data were used to locate a 159 straight segment on the right MCA M1 segment with the least curvature. The single-slice PC-MRI 160 and T1-weighted data were then acquired orthogonally to the axis of the selected MCA segment. 161 The T1-weighted data were acquired sequentially in order to monitor the changes in MCA 162 diameter.

\section{Data analysis}

164 Data analysis was carried out offline using custom R scripts (RStudio; v. 2020), GraphPad (V.8), 165 and LabChart Pro (v.8, ADInstruments, Dunedin, Otago, New Zealand).

\section{Transcranial Doppler ultrasound}

167 The MCA blood velocity (via TCD) and MAP were averaged beat-by-beat over the cardiac cycle 168 then were exported at $5 \mathrm{~Hz}$ sampling frequency and saved as text files (LabChart Pro v.8, 169 ADInstruments, Dunedin, Otago, New Zealand). During the TCD collection phase of testing, start 170 and end times of each event throughout the experimental breathing protocol were chronicled by 171 comments added in the LabChart file.

\section{Magnetic resonance imaging (MRI; 7 Tesla)}

173 During the MRI collection phase of testing, start and end times of each event were chronicled

174 based on the time associated with the desired $\mathrm{PETCO}_{2}$ on the exported RespirAct ${ }^{\mathrm{TM}}$ data file. For 175 the T1-weighted anatomical MCA images, start and end times for each protocol event were 176 recorded based off the MRI console such that images were lined up offline based on the DICOM 177 image acquisition time. The brain anatomical images were imported into a DICOM reader, OsiriX 178 software (Pixmeo@, Bernex, Switzerland), and MCA cross-sectional area (CSA) was measured by 179 a blinded rater (MK) and compared against an expert rater (BKA).

180 The PC-MRI data were acquired for 30 seconds at baseline and within a $\sim 70$-second window 181 following 50-60 seconds from the start of steady-state hypercapnia (as noted on Fig 4, panel J with 182 the PC-MRI text bar). The MCA blood velocity measurements during the MRI session were 183 obtained from PC-MRI data by manually contouring a region-of-interest (ROI) inside the MCA 
Middle cerebral artery responses to vasoreactivity protocols

184 lumen. The contours were drawn using the software, signal processing in NMR (SPIN-Research,

185 MR Innovations Inc., Detroit, MI, USA). Care was taken to avoid any peripheral voxels within the

186 MCA lumen. The magnitude PC-MRI data was used to locate the MCA lumen. The peak velocities

187 were calculated for each subject for baseline and hypercapnic states.

\section{Data extraction}

189 The LabChart text files, the TCD and MRI session RespirAct ${ }^{\mathrm{TM}}$ breath-by-breath $\mathrm{PETCO}_{2}$ values,

190 and the measured CSA were aligned using RStudio (v. 2020) (14) for data extraction from specific

191 epochs as indicated by the event comments in each file. The "print" function in the "magicfor"

192 package in $\mathrm{R}$ (15) was used to extract data with each loop iteration for each participant, and

193 variables of interest were exported as .csv files and imported to GraphPad Prism for graphing and

194 analysis.

195 Steady-state condition time courses were baseline corrected by subtracting mean baseline value (-

19630 to 0 seconds of time window of interest) for each respective variable and plotted as delta values

197 (Fig. 4). For the baselines and steady-state condition, the data were extracted from the following 198 sections within the protocol: 1) 30 seconds baseline prior to onset of hypocapnia prior to the ramp 199 protocol and 2) 30 seconds baseline prior to steady-state hypercapnia, and 3) 60 seconds at the end 200 of steady-state hypercapnia. The ramp slope analysis included the entire hypocapnic to 201 hypercapnic incremental data (see below).

\section{Ramp protocol}

203 The target $\mathrm{PETCO}_{2}$ and ramp protocol schematic is shown in Fig. 1, panel a. The target ventilation

204 rate was 12 breaths/minute and participants were coached using a visual metronome. In order to 205 equalize the spacing on the time axis when plotting the achieved $\mathrm{PETCO}_{2}$ data, the $\mathrm{PETCO}_{2}$ and 206 corresponding time vectors were resampled to a fixed 12 breaths/minute sampling rate using the 207 base R “approx" function in RStudio. Thus, two time vectors were created: 1) a target time vector 208 that is based off of a 12 breath/minute ventilation rate and 2) a "fixed" time vector that is based 209 off of resampling each participants data to meet the target time vector. The inter-individual 210 differences for the "fixed" time vector are indicated by horizontal error bars (mean \pm S.D.; Fig. 3, 211 panels a-b), and the data are plotted and averaged for all participants along the target 12 212 breaths/minute time vector. 
Middle cerebral artery responses to vasoreactivity protocols

213 MCA blood velocity and systemic MAP data (in 0.2 second increments or $5 \mathrm{MHz}$ sampling 214 frequency) were plotted from the hypocapnic state to the hypercapnic state during the ramp 215 protocol and averaged at each time point across the 12 participants (Fig. 3 panels c-d). Similarly,

216 the CSA values along the ramp protocol were averaged for each time point across participants

217 (Fig. 3 panel e). Although our goal was to collect 4 minutes of ramp data, the transition point from

218 the nadir of hypocapnia to start of ramp was different for each person and it generally took

219 approximately 2 breaths ( $\sim 12$ seconds) to sync with the desired $\mathrm{PETCO}_{2}$ for the hypercapnic ramp.

220 Thus, to ensure the same number of samples $(\mathrm{n}=12)$ for each time point along the ramp protocol,

221 we only extracted the last 228 seconds of ramp data for all participants (instead of the full 240

222 seconds).

\section{Steady-state protocol}

224 Data extraction and organization were similar to the ramp protocol except data extraction occurred

225 between the start and end of the three-minute steady-state hypercapnia stimulus. As previously 226 mentioned, the PC-MRI data acquisition commenced 50-60 seconds from start of steady-state 227 hypercapnia. Thus, the continuous T1-anatomical MCA CSA scans were interrupted to allow for 228 PC-MRI imaging (correlation with TCD measures of blood velocity are shown in Fig. 2). PC-MRI 229 data were collected for 11 out of our 12 participants. As there were shifts in PC-MRI data collection 230 start and end times across participants, the upper and lower bounds of these time points are 231 indicated under the "PC-MRI" text bar on Fig. 3 (panel e, bottom row) to indicate to the reader 232 that the mean and S.D. for CSA values in this portion of the protocol do not include all 11 233 participants (i.e., $\mathrm{n}<11)$ for the CSA data.

234 The averaged raw values for the 30 seconds baseline (prior to start of stead-state hypercapnia; 235 indicated as B on X-axis in Fig. 4) and last minute of steady-state hypercapnia (indicated as SSHC 236 on x-axis on Fig. 4) are shown in Fig. 4 for $\mathrm{PETCO}_{2}, \mathrm{MCA}$ blood velocity (via TCD) and mean 237 arterial pressure (MAP) during the TCD session and the PETCO, MCA blood velocity (via PC238 MRI) and MCA CSA during the MRI session.

MCA blood velocity reactivity calculations

240 The ramp and steady-state hypercapnia MCA blood velocity cerebrovascular reactivity (CVR) 241 measures are shown in Fig. 5 panel a. For the ramp protocol, the MCA blood velocity slope (Fig. 
Middle cerebral artery responses to vasoreactivity protocols

3 panel c) and the $\mathrm{PETCO}_{2}$ (for TCD session; Fig. 3 panel a) slope were calculated for each person

243 and the MCA blood velocity CVR was calculated as:

$$
\text { MCA Blood Velocity CVR }=\frac{\text { MCA Blood Velocity Slope }}{\mathrm{PETCO}_{2} \text { Slope }}
$$

245 For the steady-state hypercapnia protocol, the difference between the average baseline before start of hypercapnia and the average of the last minute of hypercapnia (i.e., the difference between the SSHC and B conditions in Fig. 4) were calculated. For each individual, the MCA blood velocity

248 CVR during the steady-state hypercapnia condition was then calculated as:

$$
\text { MCA Blood Velocity CVR }=\frac{\Delta \mathrm{MCA} \text { Blood Velocity }}{\Delta \mathrm{PETCO}_{2}}
$$

\section{MCA blood flow reactivity calculations}

251 The ramp and steady-state hypercapnia MCA blood flow CVR measures are shown in Fig. 5 panel

252 b. For the ramp protocol, the MCA blood velocity was sectioned into 14 second averages 253 corresponding to each CSA image along the ramp protocol. Each of these averaged MCA blood 254 velocity values were multiplied by the corresponding CSA for the given time point to calculate 255 blood flow at 14 second increments along the ramp protocol. To keep it consistent with the MCA 256 blood velocity CVR measures, the $\mathrm{PETCO}_{2}$ slopes from the TCD sessions were used as the 257 denominator during CVR calculations. The slopes were calculated for each person and the MCA 258 blood flow CVR was calculated for each individual as:

$$
\text { MCA Blood Flow CVR }=\frac{\text { MCA Blood Flow Slope }}{\mathrm{PETCO}_{2} \text { Slope }}
$$

260 Finally, to compare the MCA blood velocity CVR values and the MCA blood flow CVR values 261 calculated during the steady-state hypercapnia protocol, the percentage change from baseline for each of the MCA blood velocity or blood flow were calculated (Fig. 5, panel c).

\section{Statistical summary}

264 Inter-rater variability was assessed using Bland-Altman analysis for 45 randomly selected images.

265 Pearson's correlation coefficient was used to test the correlation for inter-modality (TCD vs. PC266 MRI; Fig. 2) MCA blood velocity measures for both baseline and steady-state hypercapnia states 267 ( $\mathrm{P}<0.05$ was considered to be statistically significant using a two-tailed test). In addition, linear 268 slope analysis was conducted to assess changes in $\mathrm{PETCO}_{2}, \mathrm{MAP}, \mathrm{MCA} \mathrm{CSA}$, and MCA blood 
Middle cerebral artery responses to vasoreactivity protocols

269

270

271

272

273

274

275

276

277

278

279

280

281

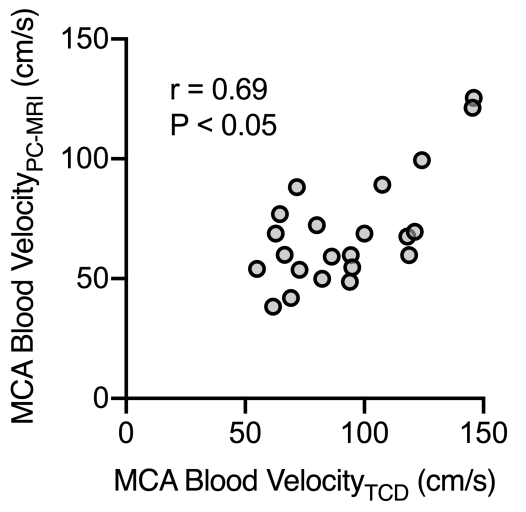

\section{Results}

Our CVR protocols with target $\mathrm{PETCO}_{2}$ are illustrated in Fig. 1. Inter-rater variability for CSA measures using the Bland-Altman test indicated a bias of $0.15 \pm 0.26 \mathrm{~mm}^{2}$ (mean \pm S.D.; BKA $\mathrm{MK}$ ) and 95\% Limits of Agreement from -0.37 to $0.67 \mathrm{~mm}^{2}$ (45 randomly selected images). Intermodality (TCD vs PC-MRI) correlation for MCA blood velocity measures indicated a significant Pearson correlation between baseline and steady-state hypercapnia for each modality $(\mathrm{n}=11$ participants, 22 pairs; $\mathrm{r}=0.69, \mathrm{P}<0.05$; Fig. 2).

\footnotetext{
Figure 2 - MCA blood velocity measure comparison between TCD and PC-MRI. Correlation plot comparing MCA blood velocity measured from transcranial Doppler (TCD) and phase contrast magnetic resonance imaging (PC-MRI). Baseline measures for both TCD and MRI sessions and steady-state hypercapnia measure at the $\sim 1$ 2 minute mark for PC-MRI and in the last minute of steady-state hypercapnia for the TCD session for 22 pairs $(\mathrm{n}=11 ; \mathrm{r}=0.69, \mathrm{P}<0.05)$.
}

282 Slope analysis during the ramp protocol indicated significant slopes $(\mathrm{P}<0.05$; Fig. 3 panels a - d) 283 for achieved $\triangle \mathrm{PETCO}_{2}(\mathrm{mmHg})$ during $\mathrm{TCD}\left(\mathrm{Y}=0.033 * \mathrm{X}-3.96 ; \mathrm{R}^{2}=0.88\right)$ and $\mathrm{MRI}(\mathrm{Y}=$ $\left.2840.027 * \mathrm{X}-2.54 ; \mathrm{R}^{2}=0.77\right)$ sessions, with corresponding slopes for $\Delta \mathrm{MCA}$ blood velocity $(\mathrm{cm} / \mathrm{s}$; $\left.285 \mathrm{Y}=0.13 * \mathrm{X}-13.70 ; \mathrm{R}^{2}=0.59\right)$ and $\Delta \mathrm{MAP}\left(\mathrm{mmHg} ; \mathrm{Y}=0.009 * \mathrm{X}-0.38 ; \mathrm{R}^{2}=0.02\right)$. Although 286 significant, the slope for MAP during the ramp protocol indicated an average $<2 \mathrm{mmHg}$ increase 287 throughout the protocol. The slope of the MCA CSA with time did not show a deviation from 0 across the ramp protocol (Fig. 3, panel E; Y $=0.0008 * \mathrm{X}-0.057 ; \mathrm{R}^{2}=0.006$ ). 
Middle cerebral artery responses to vasoreactivity protocols
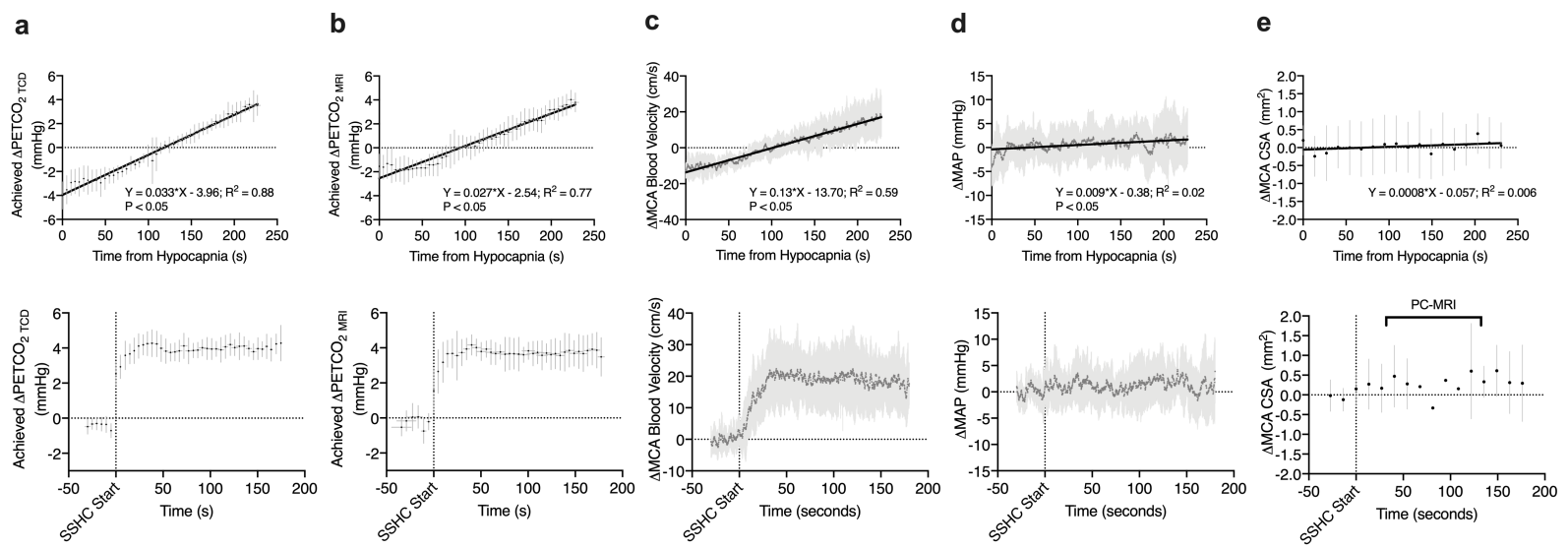

Figure 3 - Measured variables during the ramp and steady-state hypercapnia protocols. Ramp protocol (228 seconds; top row) responses and steady-state hypercapnia (180 seconds; bottom row) for delta changes in each variable from baseline levels are shown. Panel a: achieved $\mathrm{PETCO}_{2}$ for TCD session, panel b: achieved $\mathrm{PETCO}_{2}$ for MRI session, panel c: MCA blood velocity in TCD session, panel d: mean arterial pressure (MAP), and panel e: MCA cross-sectional area (CSA). The "PC-MRI" text on panel e bottom row indicates the variable time window in which PC-MRI images were acquired and there was an interruption in the consistent MCA CSA measurements. A 30 second baseline is shown for the steady-state hypercapnia condition (bottom row) and hypercapnia is indicated by SSHC on x-axis with a vertical line. Linear regressions (panels a-e top row) are shown for each variable in the ramp protocol and a significant (non-zero; $\alpha$ level significance 0.05 ) slope is indicated by $\mathrm{P}<0.05 . \mathrm{N}=12$ for all variables with data presented as mean \pm S.D.

289 During the steady-state hypercapnia protocol, $\mathrm{PETCO}_{2}$ increased during steady-state hypercapnia

290 from baseline with a mean difference of $4.45 \mathrm{mmHg}$ and $95 \%$ CI of 4.05 to 4.85 when using TCD

291 (44.3 \pm 3.1 vs. $39.9 \pm 2.9 \mathrm{mmHg}$, respectively; $\mathrm{n}=12, \eta_{\mathrm{p}}{ }^{2}=0.98, \mathrm{P}<0.05$; Fig. 4, panel a) and a mean 292 difference of 3.75 and $95 \%$ CI of 3.29 to 4.20 when using MRI (42.7 \pm 4.2 vs. $38.9 \pm 4.4 \mathrm{mmHg}$, 293 respectively; $\mathrm{n}=12, \eta_{\mathrm{p}}{ }^{2}=0.97, \mathrm{P}<0.05$; Fig. 4 , panel b) sessions. Similarly, MCA blood velocity 294 increased with steady-state hypercapnia from baseline with a mean difference of $18 \mathrm{~cm} / \mathrm{s}$ and $95 \%$ $295 \mathrm{CI}$ of 13 to $24 \mathrm{~cm} / \mathrm{s}$ in the TCD trial $\left(104 \pm 29\right.$ vs. $86 \pm 24 \mathrm{~cm} / \mathrm{s}$, respectively; $\mathrm{n}=12, \eta_{\mathrm{p}}{ }^{2}=0.83, \mathrm{P}<0.05$;

296 Fig. 4, panel c) and a mean difference of $22 \mathrm{~cm} / \mathrm{s}$ and 95\% CI of 12 to 33 when using MRI (via 297 PC-MRI; $79 \pm 15$ vs. $59 \pm 9 \mathrm{~cm} / \mathrm{s}$, respectively; $\mathrm{n}=11, \eta_{\mathrm{p}}{ }^{2}=0.70, \mathrm{P}<0.05$; Fig. 4 panel e) sessions, and 298 MCA CSA increased with a mean difference of $0.31 \mathrm{~mm}^{2}$ and $95 \% \mathrm{CI}$ of -0.03 to $0.66 \mathrm{~mm}^{2}$ during 299 the MRI session (5.71 \pm 1.03 vs. $5.34 \pm 0.97 \mathrm{~mm}^{2}$, respectively; $\mathrm{n}=12, \eta_{\mathrm{p}}{ }^{2}=0.27, \mathrm{P}<0.05$; Fig. 4, 300 panel $\mathrm{f}$ ). The mean difference in MAP between the last minute of steady-state hypercapnia and 301 baseline was $1.35 \mathrm{mmHg}$ with a $95 \% \mathrm{CI}$ of -0.82 to $3.5 \mathrm{mmHg}$ which is most compatible with a 302 negligible change ( $90 \pm 8$ vs. $89 \pm 8 \mathrm{mmHg}$, respectively; $\mathrm{n}=12 ; \eta_{\mathrm{p}}{ }^{2}=0.14$, Fig. 4 , panel e). Calculated MCA blood flows (product of MCA blood velocity and CSA) increased from baseline to steady- 
Middle cerebral artery responses to vasoreactivity protocols

state hypercapnia ( $267 \pm 54$ vs. $348 \pm 78 \mathrm{ml} / \mathrm{min}$, respectively; $\mathrm{P}<0.05$, one-tailed paired t-test) with a delta calculated blood flow of $81 \pm 30 \mathrm{ml} / \mathrm{min}$.
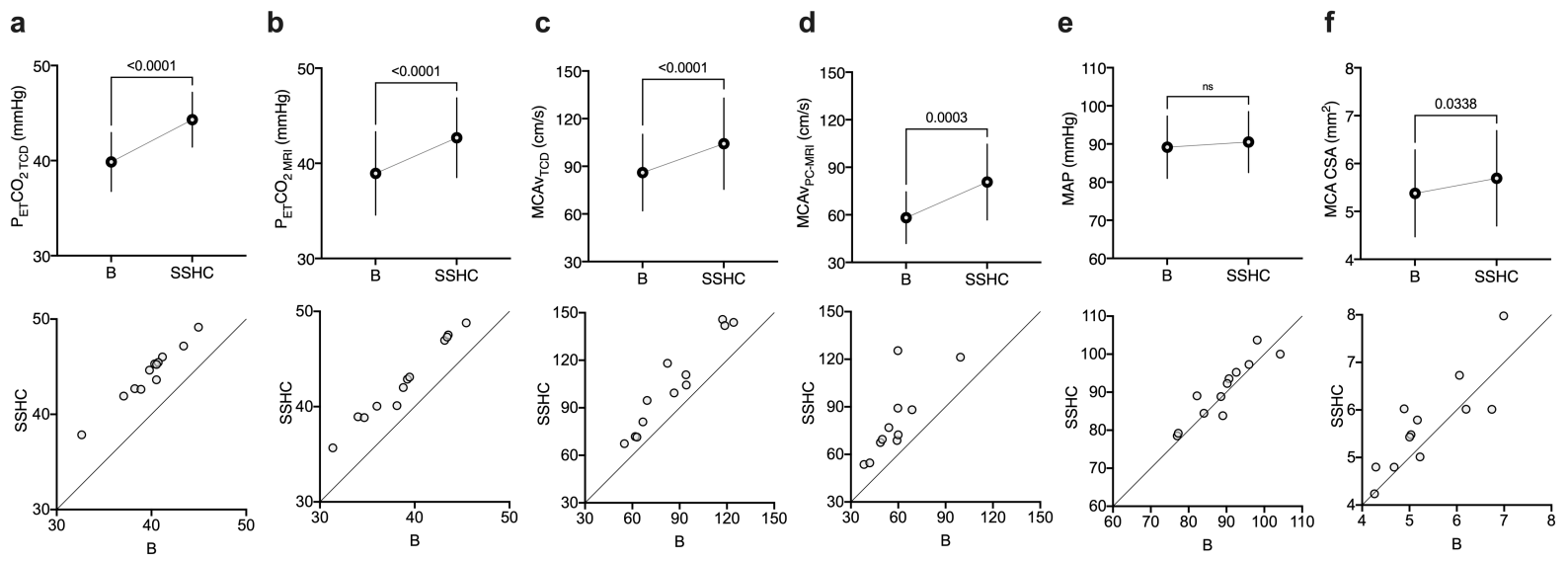

Figure 4 - Baseline (B) and steady-state hypercapnia (SSHC) comparisons. Top row: Baseline (B) and steady-state hypercapnia (SSHC) measures ( $n=12$ except for panel $d$ where $n=11$, mean \pm S.D.). Where statistical significance occurs, $\mathrm{p}$-values are indicated above data ( $\mathrm{ns}=$ not significant; paired ttest; $\alpha$ level significance 0.05$)$. Bottom row: Individual data points $(\mathrm{n}=12$ except for panel $\mathrm{d}$ where $\mathrm{n}=11)$ comparing baseline (B) to steady-state hypercapnia (SSHC) responses for the same variable in that column as top row. The diagonal line (identity line) indicates where data would fall if there was no measurable effect of SSHC from B. Data above the identity line indicates an increase in variable measure with SSHC (from baseline; B).

The mean difference in MCA blood velocity-based measure of CVR between the ramp and steadystate hypercapnia protocols was 0.18 with $95 \%$ CI of -0.59 to 0.96 which is most compatible with a negligible effect of protocol on MCA blood velocity CVR $(3.8 \pm 1.7$ vs. $4.0 \pm 1.6 \mathrm{~cm} / \mathrm{s} / \mathrm{mmHg}$, respectively; $n=12$, two-tailed paired t-test, $\mathrm{p}=0.62, \eta_{\mathrm{p}}{ }^{2}=0.02$, Fig. 5, panel a). The mean difference in calculated MCA blood flow-based measure of CVR between the steady-state protocol and ramp

312 protocol was 5.0 with a $95 \%$ CI of 2.8 to $7.2 \mathrm{ml} / \mathrm{min} / \mathrm{mmHg}$ and was statistically significant

$313\left(17.3 \pm 5.7\right.$ vs. $12.3 \pm 4.5 \mathrm{ml} / \mathrm{min} / \mathrm{mmHg}$, respectively; $\mathrm{n}=12$, two-tailed t-test, $\mathrm{P}<0.05, \eta_{\mathrm{p}}{ }^{2}=0.70$,

314 two-tailed t-test; Fig. 5, panel b). Similarly, when calculated as \%change in MCA blood velocity

315 or MCA blood flow over the $\triangle \mathrm{PETCO}_{2}$ during hypercapnia, the mean difference between MCA

316 blood flow CVR and MCA blood velocity CVR was $(6.7 \pm 1.6$ vs. $4.8 \pm 2.0 \% / \mathrm{mmHg}$, respectively;

$317 \mathrm{n}=12, \mathrm{P}<0.05$, one-tailed t-test). Finally, the \%change of MCA blood flow was greater than the

$318 \%$ change of MCA blood velocity during the steady-state condition with a mean difference of 6.9

319 and $95 \%$ CI of -0.37 to 14.10 (29.8 \pm 8.2 vs. $21.5 \pm 9.6 \%$, respectively; $n=12, \mathrm{P}<0.05$, one-tailed t-

320 test; Fig. 5, panel c). 
Middle cerebral artery responses to vasoreactivity protocols
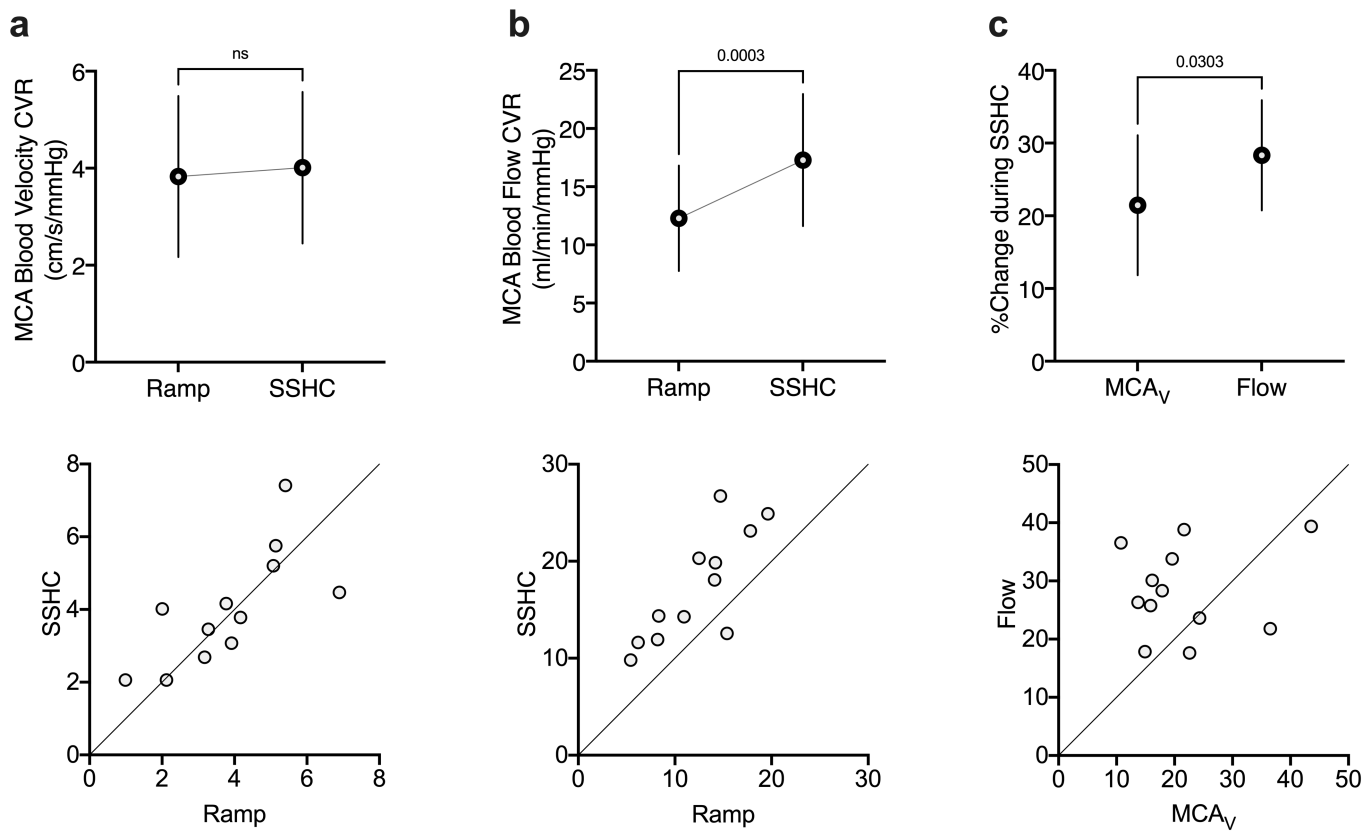

Figure 5 - Middle cerebral artery (MCA) blood velocity and flow reactivity across protocols Top row: Calculated MCA blood velocity cerebrovascular reactivity (CVR) was not different between ramp and steady-state hypercapnia (SSHC; panel a), while MCA blood flow CVR was higher in SSHC compared to the ramp protocol (panel b). The percent change in calculated MCA blood flow from baseline was higher than the percent change in MCA blood velocity from baseline (panel c) with SSHC. Where statistical significance occurs, $\mathrm{p}$-values are indicated above data ( $\mathrm{ns}=$ not significant; paired t-test; $\alpha$ level significance 0.05$)$. $\mathrm{N}=12$ for all variables with data presented as mean \pm S.D. Bottom row: Individual data points $(n=12)$ comparing ramp to steadystate hypercapnia (SSHC) responses (panels a-b) for the same variable in that column as top row or MCA blood velocity to calculated MCA blood flow (panel c). The diagonal line (identity line) indicates where data would fall if there was no measurable effect of protocol on CVR measures (panels a-b) or effect of accounting for MCA CSA in flow calculations (compared to using MCAv alone as an index of flow) when assessing \%change of flow or MCAv during SSHC (panel c). Data above the identity line indicates an increase in variable measure with SSHC (compared to ramp; panels a-b) or higher \%change in flow value for a given \%change in MCAv.

\section{Discussion}

322 This is the first study to provide MCA CSA measures across ramp and steady-state hypercapnia

323 protocols, enabled by the ability to obtain MCA CSA measurements every 14 seconds with

324 prospective targeting of $\mathrm{PETCO}_{2}$. This approach enabled direct comparisons of the steady- state

325 versus a ramp protocols to establish valid CVR calculations using only measures of flow velocity.

326 The noteworthy findings of this study are that: 1) the MCA CSA did not change during the ramp

327 protocol with delta $\sim-3$ to $+4 \mathrm{mmHg}$ of $\mathrm{PETCO}_{2}$, but did increase with steady-state hypercapnia 
Middle cerebral artery responses to vasoreactivity protocols

$\sim+4 \mathrm{mmHg}$ of $\mathrm{PETCO}_{2}$ ), 2) blood pressure remained stable throughout duration of both ramp and steady-state protocols, and 3) CVR measures based on MCA blood velocity cerebrovascular reactivity was not different between ramp and steady-state protocols, but 4) MCA blood flowbased CVR was greater during steady-state compared to the ramp protocol. Taken together, the ramp protocol seems to result in similar CVR values as those observed in the steady-state protocol, with the added advantage of having minimal and negligible effects on MCA CSA or MAP in the face of moderate elevations in $\mathrm{PETCO}_{2}$.

\section{Transcranial Doppler - MCA blood velocity \& CVR measures}

Often, CVR is characterized by the slope in the linear relationship between MCA blood velocity and $\mathrm{PETCO}_{2}$ with respect to each variable's relative change with time. The relationship between arterial blood velocity and $\mathrm{CO}_{2}(16)$ is sigmoidal and both the range and starting point of $\mathrm{PETCO}_{2}$ affect the arterial blood velocity response to $\mathrm{CO}_{2}$ (17). Regan et al. (7) showed a lower MCA blood velocity-based CVR during steady-state hypercapnia $\left(\triangle \mathrm{PETCO}_{2}\right.$ of $\left.10 \mathrm{mmHg}\right)$ than during the ramp hypercapnic protocol ( -5 to $+10 \mathrm{mmHg} \mathrm{PETCO}_{2}$ ). However, these ranges of $\mathrm{PETCO}_{2}$ can elicit systemic hemodynamic effects (6) that can increase blood velocity changes independent of cerebral vascular bed dilation. The current protocols used a lower dose of change in $\mathrm{PETCO}_{2}$ in order to avoid the central hemodynamic and non-linear portions of the CVR curve.

\section{MRI-MCA CSA \& CVR measures}

In the current study, $\mathrm{PETCO}_{2}$ values at the ends of both ramp and steady-state hypercapnia protocols, and the calculated MCA blood velocity CVR measures, were not different between the two protocols. Yet, the $\mathrm{PETCO}_{2}$ changes during the ramp protocol did not affect MCA CSA in same the way that the steady-state hypercapnia protocol did. To quantify transient MCA CSA measures, we developed an anatomical scan optimized to provide MCA images every 14 seconds. This enabled imaging of the MCA during the dynamic stimuli such as the ramp protocol or onset of the steady state protocol, as well as improving temporal sensitivity during the steady-state model. To our knowledge, our 14 second anatomical scan of MCA CSA provides the highest level of temporal resolution (i.e., shortest acquisition time) available when compared to existing assessments of MCA CSA during steady-state hypercapnia protocols. From our current MCA CSA findings, the MCA dilates under conditions where hypercapnia is elevated and sustained (i.e. steady-state hypercapnia), rather than with brief exposure to elevated levels of $\mathrm{PETCO}_{2}$ (i.e., end 
Middle cerebral artery responses to vasoreactivity protocols

of ramp protocol). This observation is supported by previous studies indicating a slow onset to dilation in either the internal carotid artery (18) or the MCA (2), despite immediate changes in MCA blood velocity that reflect downstream microvascular dilation to early hypercapnia.

This study supports previous MCA CSA findings in CVR studies that indicate MCA CSA dilates after $\sim 2$ minutes of steady-state hypercapnia using 3T (2) and 7T MRI $(4,5)$. Thus, CVR values during the steady-state protocol indicated significant error when the MCA cross-sectional area was not included. Specifically, we found that the \%change in MCA blood velocity was lower than the \%change in MCA blood flow by $7 \%$ (mean difference between these two measures). This value is less than the $18 \%$ observed in work by Coverdale et al. (2). We believe the discrepancy in these mean differences is due to the magnitude of hypercapnia achieved in each study, with $\sim \Delta 4 \mathrm{mmHg}$ $\mathrm{PETCO}_{2}$ in the current study versus $\sim+\Delta 10 \mathrm{mmHg}$ in $\mathrm{PETCO}_{2}$ for the study by Coverdale et al. As well, there was a $5 \%$ increase in MAP in the study by Coverdale et al. ( $4 \mathrm{mmHg})$ which was greater than the increase in MAP for our study $(\sim 2 \mathrm{mmHg}$ for ramp, and $\sim 1 \mathrm{mmHg}$ for steadystate), a difference that may be attributed to the greater magnitude of the hypercapnia stimulus in the the previous study. While mild variations in MAP are accounted for by autoregulatory mechanisms such that MCA blood flow is sustained with negligible influence on MCA CSA, autoregulation mechanisms are impaired during hypercapnia (19). Thus, we anticipate that the higher the hypercapnic magnitude, the greater the risk that MAP will influence MCA CSA (and/or blood velocity) augmenting any discrepancies between MCA blood velocity and calculated MCA blood flow.

This is the first study to use a sequential gas delivery circuit (via the RespirAct ${ }^{\mathrm{TM}}$ ) when assessing MCA CSA responses during hypercapnia, thereby more closely aligning partial pressure of arterial and end-tidal $\mathrm{CO}_{2}$ levels (20). Interestingly, we achieved similar MCA blood flow CVR during steady-state hypercapnia as previous work (2), and our MCA blood velocity CVR for both the steady-state hypercapnia $(2,21)$ and ramp protocol (7) were in agreement with previous studies. As recommended by Regan et al. (7), when using a limited range of $\mathrm{PETCO}_{2}$ values during a hypercapnic protocol, a linear approach to CVR analysis is appropriate.

Everything considered, a CVR measure based solely on TCD-acquired MCA blood velocity measures during a ramp hypercapnia protocol seems to elicit a similar CVR outcome as the commonly used steady-state protocol, but without the limitation of potential changes in MCA 
Middle cerebral artery responses to vasoreactivity protocols

CSA. CVR outcome measures involve calculating MCA blood velocity changes for a given change in $\mathrm{PETCO}_{2}$. Similarly, our study illustrates that even modest values of change in $\mathrm{PETCO}_{2}$ achieve a comparable value of CVR at higher hypercapnic doses.

\section{Methodological Considerations}

392 Our overall target range for manipulating $\mathrm{PETCO}_{2}$ during the cerebrovascular reactivity was

393 between the $-\Delta 5$ to $+\Delta 5$ from baseline $\mathrm{PETCO}_{2}(10)$. While our findings support a lack of MCA

394 CSA changes during ramp protocols of $-\Delta 3$ to $+\Delta 4 \mathrm{mmHg}$ in $\mathrm{PETCO}_{2}$, we cannot make conclusive remarks on MCA CSA changes during a ramp protocol of $\pm \Delta 5 \mathrm{mmHg}$ in $\mathrm{PETCO}_{2}$. However, as mentioned above, our values of $\triangle \mathrm{PETCO}_{2}$ fell within our target $\pm \Delta 5 \mathrm{mmHg}$ from baseline $\mathrm{PETCO}_{2}$ range, the CVR measures are consistent with previous studies where higher levels of $\mathrm{PETCO}_{2}$ were used, and we found negligible increases in MAP. Therefore, the modest level of $\mathrm{PETCO}_{2}$ achieved here appear to have achieved the major objectives. The ramp protocol designed for the current study also achieved an optimal balance between stimulus and central hemodynamics. We acknowledge however, that we did not account for the impact of the $\sim 2 \mathrm{mmHg}$ rise in MAP during the ramp protocol, which we expect to be negligible in impacting MCA CSA.

403 Although we were unable to measure continuous blood pressure and MCA blood velocity during 404 the MRI trial, we measured blood velocity data during the MRI session via the PC-MRI sequence. 405 Testing of the TCD and MRI segments of the study were collected consecutively within a 2-hour 406 window with the order of tests varied across participants. The absolute values for MCA blood 407 velocities measured by PC-MRI were lower than our TCD measures, although we suspect this had 408 to do with our pre-set Venc value choice of $100 \mathrm{~cm} / \mathrm{s}$ which may have cut off some of the higher 409 velocities. Our rationale for not choosing a higher Venc than $100 \mathrm{~cm} / \mathrm{s}$ was the possibility of cutting 410 off lower velocity values during baseline. Regardless, the increase in MCA blood velocity during 411 hypercapnia (from baseline) were in agreement between the two techniques (TCD: $\sim 18 \mathrm{~cm} / \mathrm{s}$ and 412 PC-MRI: $\sim \Delta 20 \mathrm{~cm} / \mathrm{s}$ ). Thus, we assume that the blood pressure responses during the TCD and 413 MRI sessions were similar as well.

414 Finally, the current results are delimited to young healthy adults Thus, additional studies are 415 needed to understand the effects of age, a group that demonstrates greater MAP responses to 416 hypercapnia and variable responses in CSA changes (9), or other differentiating conditions. 
Middle cerebral artery responses to vasoreactivity protocols

417 Further, our study was conducted in the supine position, which is less replicable for CVR studies

418 than the seated position (22) and may explain some of the disparity of blood velocity measures for

419 some individuals between the two protocols: the supine posture is necessary for MRI studies.

420 Additional studies are required to address the impact of CSA changes and protocol model on

421 posture-dependent intra-subject variability so that reliable CVR protocols can be used to make

422 inferences on vascular health (e.g., inferred vascular dysfunction with reduced CVR). The current

423 data suggest that the ramp protocol might be useful in reducing inter-study variability.

\section{Conclusions}

425 A constant CSA during experimental vasoreactive challenges is essential to the reliability of TCD-

426 acquired blood velocity as a correlative index of blood flow changes. In this study, we showed

427 data that were most compatible with negligible change in MCA CSA from baseline during a graded

428 ramp hypercapnic protocol $\left( \pm \sim \Delta 4 \mathrm{mmHg}\right.$ from baseline $\left.\mathrm{PETCO}_{2}\right)$. Similar to our previous work,

429 the MCA data align best with an interpretation of MCA dilation during a prolonged (3-minutes)

$430 \sim \Delta 4 \mathrm{mmHg}$ in baseline $\mathrm{PETCO}_{2}$ period of hypercapnia, and confirmed the expected error in

431 \%change of MCA blood velocity as an index of blood flow and in the calculated CVR when a

432 change in CSA is not considered (2). Combined, these data suggest that during transient changes

433 in $\mathrm{PETCO}_{2}$, as in the ramp protocol, the constant segment of the sigmoid may describe MCA CSA

434 changes with $\mathrm{PETCO}_{2}$, and any changes in blood velocity can reflect CBF. In summary, the ramp

435 protocol with $\pm \sim \Delta 4 \mathrm{mmHg}$ from baseline $\mathrm{PETCO}_{2}$ appears to provide expected measures of CVR

436 where blood velocity should reflect blood flow patterns of change.

\section{Acknowledgements}

438 The authors would like to thank the participants for their time, Joseph S. Gati and Trevor Szekeres

439 for their MRI expertise, Arlene Fleischhauer, and Emilie Woehrle and Jenna Schulz for helping

440 with participant recruitment. This study was funded by the Canadian Institutes of Health Research

441 (grant \# 201503MOP-342412-MOV-CEEA). KN was supported by a research grant from

442 Children's Health Foundation. BKA was funded by the MITACS Postdoctoral Elevate Fellowship.

443 JKS and RSM are Tier 1 Canada Research Chairs. 
Middle cerebral artery responses to vasoreactivity protocols

\section{Author contribution statement}

445 BKA conceptualized and designed the study, collected, analyzed, and disseminated the data, and

446 wrote and edited the manuscript. SB assisted with study design, data collection and analysis, and

447 assisted with writing and editing the manuscript. MK assisted with data collection, analysis and 448 interpretation and editing of the manuscript. BJM assisted with data collection, organization, and

449 editing the manuscript. KN assisted with study design and editing of the manuscript. RSM assisted 450 with study design and editing of the manuscript. JKS conceptualized and designed the study, and 451 assisted with data dissemination, writing and editing of the manuscript.

\section{Conflict of interest}

453 The authors do not have any conflicts of interest to disclose. 
bioRxiv preprint doi: https://doi.org/10.1101/2021.03.11.434655; this version posted March 12, 2021. The copyright holder for this preprint (which was not certified by peer review) is the author/funder, who has granted bioRxiv a license to display the preprint in perpetuity. It is made available under aCC-BY 4.0 International license.

Middle cerebral artery responses to vasoreactivity protocols

\section{References}

462

463

464

465

466

467

468

469

470

471

472

473

474

475

476

477

1. Portegies MLP, de Bruijn RFAG, Hofman A, Koudstaal PJ, Ikram MA. Cerebral vasomotor reactivity and risk of mortality: the Rotterdam Study. Stroke. 2014 Jan;45(1):42-7.

2. Coverdale NS, Gati JS, Opalevych O, Perrotta A, Shoemaker JK. Cerebral blood flow velocity underestimates cerebral blood flow during modest hypercapnia and hypocapnia. J Appl Physiol Bethesda Md 1985. 2014 Nov 15;117(10):1090-6.

3. Howe CA, Caldwell HG, Carr J, Nowak-Flück D, Ainslie PN, Hoiland RL. Cerebrovascular reactivity to carbon dioxide is not influenced by variability in the ventilatory sensitivity to carbon dioxide. Exp Physiol. 2020;105(5):904-15.

4. Al-Khazraji BK, Shoemaker LN, Gati JS, Szekeres T, Shoemaker JK. Reactivity of larger intracranial arteries using 7 T MRI in young adults. J Cereb Blood Flow Metab Off J Int Soc Cereb Blood Flow Metab. 2019 Jul;39(7):1204-14.

5. Verbree J, Bronzwaer A-SGT, Ghariq E, Versluis MJ, Daemen MJAP, van Buchem MA, et al. Assessment of middle cerebral artery diameter during hypocapnia and hypercapnia in humans using ultra-high-field MRI. J Appl Physiol Bethesda Md 1985. 2014 Nov 15;117(10):1084-9.

6. Shoemaker JK, Vovk A, Cunningham DA. Peripheral chemoreceptor contributions to sympathetic and cardiovascular responses during hypercapnia. Can J Physiol Pharmacol. 2002 Dec;80(12):1136-44.

7. Regan RE, Fisher JA, Duffin J. Factors affecting the determination of cerebrovascular reactivity. Brain Behav. 2014 Sep;4(5):775-88.

8. Stefanidis KB, Askew CD, Klein T, Lagopoulos J, Summers MJ. Healthy aging affects cerebrovascular reactivity and pressure-flow responses, but not neurovascular coupling: A cross-sectional study. PLOS ONE. 2019 May 16;14(5):e0217082.

9. Coverdale NS, Badrov MB, Shoemaker JK. Impact of age on cerebrovascular dilation versus reactivity to hypercapnia. J Cereb Blood Flow Metab. 2017 Jan 1;37(1):344-55.

10. Hoiland RL, Fisher JA, Ainslie PN. Regulation of the Cerebral Circulation by Arterial Carbon Dioxide. Compr Physiol. 2019 Jun 12;9(3):1101-54.

11. McKetton L, Cohn M, Tang-Wai DF, Sobczyk O, Duffin J, Holmes KR, et al. Cerebrovascular Resistance in Healthy Aging and Mild Cognitive Impairment. Front Aging Neurosci. 2019;11:79.

12. Fisher JA, Sobczyk O, Crawley A, Poublanc J, Dufort P, Venkatraghavan L, et al. Assessing cerebrovascular reactivity by the pattern of response to progressive hypercapnia. Hum Brain Mapp. 2017 Jul;38(7):3415-27. 
Middle cerebral artery responses to vasoreactivity protocols

13. Brothers RM, Lucas RAI, Zhu Y-S, Crandall CG, Zhang R. Cerebral vasomotor reactivity: steady-state versus transient changes in carbon dioxide tension. Exp Physiol. 2014 Nov;99(11):1499-510.

14. Core Team R. R: A language and environment for statistical computing. R Foundation for Statistical Computing, Vienna, Austria (2013). Suppl Fig S. 2015;2.

15. Makiyama K. magicfor: Magic Functions to Obtain Results from for Loops [Internet]. 2016 [cited 2021 Jan 9]. Available from: https:/CRAN.R-project.org/package=magicfor

16. Battisti-Charbonney A, Fisher J, Duffin J. The cerebrovascular response to carbon dioxide in humans. J Physiol. 2011 Jun 15;589(Pt 12):3039-48.

17. Sobczyk O, Battisti-Charbonney A, Fierstra J, Mandell DM, Poublanc J, Crawley AP, et al. A conceptual model for $\mathrm{CO}_{2}$-induced redistribution of cerebral blood flow with experimental confirmation using BOLD MRI. NeuroImage. 2014 May 15;92:56-68.

18. Willie CK, Macleod DB, Shaw AD, Smith KJ, Tzeng YC, Eves ND, et al. Regional brain blood flow in man during acute changes in arterial blood gases. J Physiol. 2012 Jul 15;590(14):3261-75.

19. Panerai RB, Deverson ST, Mahony P, Hayes P, Evans DH. Effects of CO2 on dynamic cerebral autoregulation measurement. Physiol Meas. 1999 Aug;20(3):265-75.

20. Ito S, Mardimae A, Han J, Duffin J, Wells G, Fedorko L, et al. Non-invasive prospective targeting of arterial $\mathrm{P}(\mathrm{CO} 2)$ in subjects at rest. J Physiol. 2008 Aug 1;586(15):3675-82.

21. Burley CV, Lucas RAI, Whittaker AC, Mullinger K, Lucas SJE. The CO2 stimulus duration and steady-state time point used for data extraction alters the cerebrovascular reactivity outcome measure. Exp Physiol. 2020 May;105(5):893-903.

22. McDonnell MN, Berry NM, Cutting MA, Keage HA, Buckley JD, Howe PRC. Transcranial Doppler ultrasound to assess cerebrovascular reactivity: reliability, reproducibility and effect of posture. PeerJ. 2013 Apr 9;1:e65. 